\title{
Electromagnetic Shielding Performance Measurement for Braided- Shield Power Cables at Low Frequency Regimes
}

\author{
Zhongyuan Zhou, Peng Hu, Xiang Zhou, Mingjie Sheng, Peng Li, Qi Zhou \\ Electromagnetic Compatibility Laboratory, School of Mechanical Engineering, Southeast University, Nanjing 211189, China, \\ hfut_hupeng@126.com
}

\begin{abstract}
Although various measurement techniques have been applied to both qualitative and quantitative evaluation for the electromagnetic shielding performance of braided-shield power cables, the existing measurements cannot directly assess the low-frequency shielding performance (typically below $100 \mathrm{kHz}$ ) due to factors such as ground-loop effects and dynamic range problem in measurement. To solve these, an improved shield reduction factor method, based on gain (T/R) rather than scattering parameters, is proposed to evaluate the shielding performance of braided-shield power cables from $25 \mathrm{~Hz}$ to $1 \mathrm{MHz}$. In this work, we highlight the implementation of measurement setup to avoid the effects of ground-loop and stray electromagnetic field. Meanwhile, the test cell is simplified according to the definition of the shield reduction factor in order to obtain the gain (T/R) parameters, which can be used to calculate transfer impedance as well. From the measurements we present more intuitive evaluation of shielding behavior of braided-shield power cables at low frequency regimes, and showcase a detailed comparative discussion between transfer impedance and shield reduction factor. The proposed shield reduction factor method is expected to be a useful way for the evaluation of the low frequency shielding performance of braided-shield cables.
\end{abstract}

Keywords: Braided-shield power cable, electromagnetic shielding performance, shield reduction factor, measurement technique.

\section{INTRODUCTION}

Braided-shield power cables play an important role in the interconnection of various high-voltage (HV) high-power (HP) electrical drives, e.g., in high-speed railway traction, electric ship propulsion, or electric vehicle drive system. Considering the wide application of non-linear, unbalanced loads and HP electronic converters, however, electromagnetic interference (EMI) inevitably leads to performance degradation of electronics, which may turn out to be insecure against the reliable operation of control system [1]. Moreover, a series of electromagnetic compatibility (EMC) experiments show that these interconnected cables are considered to account for the radiated emission problems [2], [3]. Theoretically, braided-shield cables with proper grounding provide an effective way to protect against electromagnetic emissions and couplings. The material, wire diameter and weave angle of the braided shield will affect the EMI suppression effect, and consequently qualitative and quantitative evaluation of the coupling mechanism of electromagnetic field (EMF) to the braided shield is a prerequisite for optimizing the material and geometric parameters.

In general, either transfer impedance $\left(Z_{t}\right)$ or shield reduction factor $\left(K_{r}\right)$ is the characteristic parameter used to analyze electromagnetic shielding performance of the cables, and $Z_{t}$ or $K_{r}$ can be approximated by analytical formulas [4]-[7]. According to the definitions, transfer impedance and shield re- duction factor are all positively correlated with the shielding performance, i.e., the smaller $Z_{t}$ or $K_{r}$ is, the better shielding performance of the cable will be. Different from theoretical calculation, direct measurement is the most effective way to evaluate shielding performance of braided-shield cables. Over the recent years, various methods have been developed to measure transfer impedance $Z_{t}$ of the braided-shield cables, and these methods, e.g., the triaxial method (IEC 62153-4-3) and the line injection method (IEC 62153-4-6), are mostly suitable for the metallic communication cables which share the same coaxial or quasi-coaxial characteristic structures. With regard to HV braided-shield power cables, the differences in diameter and noncoaxial structure may make the standard methods unapplicable as they are. As discussed in [8] and [9], the triaxial method must prepare different tubes or even a larger triaxial cell in order to reasonably fix some certain power cables with different geometric features. In [10], the line injection method may yield different results caused by different orientations of injection line along the noncoaxial cable under test (CUT). To overcome these, the ground plate method (GPM) had been proposed for the measurement of $Z_{t}$, and effectively solved the problem of geometric features in different samples [9], [11], [12]. It is worth noting, however, that the ground-loop in GPM (both source and receiver have the same ground potential) will affect the measurements especially at frequency below $100 \mathrm{kHz}$ (more details can be 
found in IEC 62153-4-3 Annex F), where the low frequency limit for GPM mainly relies on that of ferrites used to avoid the ground-loop effects.

Shield reduction factor $K_{r}$, compared with transfer impedance $Z_{t}$, can provide more intuitive evaluation of electromagnetic shielding performance of braided-shield cables at low frequency (typically below $1 \mathrm{MHz}$ ) [7]. In [13], a flux density probe has been used for the measurement of $K_{r}$ at 50 $\mathrm{Hz}$, and obviously this is the most direct way to assess the shielding performance for several specific frequency points. Note that the CUT in [13] is placed in the metal wiring trough, the measured $K_{r}$ represents the shielding performance of the wiring trough with and without cover, and more generally, the probe cannot be placed inside the braided shield of CUT [14]. As for the standard procedure of $K_{r}$ for communication cables in the $50-800 \mathrm{~Hz}$ regime [15], the test cell can be easily constructed in case of electromagnetic coupling between the parallel lines. However, variable-frequency power source (VFPS) and high-precision probe are all essential for the measurement configuration. As per [15] the source should be directly loaded onto the braided shield of CUT, it is uncertain how many amperes will have to be supplied by the power source to ensure that the measurement has a sufficient dynamic range, and how to avoid ground-loop effects, as well as stray EMF, on measurement signal typically below $100 \mathrm{kHz}$.

To overcome the aforementioned questions without significantly compromising the preparation time, cost and experimental results of CUT, the transmission-reflection (T/R) technique is used to measure the gain parameters rather than scattering parameters especially at frequencies below $1 \mathrm{MHz}$. In this work, the test cell is improved by simplifying the braided shield to ground loop in order to meet the requirements of geometric features for different braided-shield power cables, while large dynamic range $(\geq 90 \mathrm{~dB})$ for the measurement of gain $(T / R)$ parameters is provided by gain-phase test port of vector network analyzer (VNA).

In this paper, which is an extension of the investigation presented in [7], we highlight the shield reduction factor method (SRFM) and the equivalence principle for the shielding performance assessment of braided-shield power cables to improve the test cell which can be suitable for the measurement of gain (T/R) parameters from $25 \mathrm{~Hz}$ to $1 \mathrm{MHz}$, and finally provide intuitive evaluation for the shielding performance of CUT. This paper is structured as follows. Section 2 presents the generalized definition of shield the reduction factor, and corresponding implementation of SRFM for the measurements as an extension of the work in [7]. Section 3 shows results for shielding performance of CUT evaluated by shield reduction factor as well as transfer impedance, and also showcases a detailed comparative discussion. Finally, Section 4 summarizes the conclusions of this paper.

\section{THEORY}

\subsection{Shield reduction factor}

For two independent parallel coils, an electrified coil will induce a reverse current in another coil according to Lenz's law. Similarly as visible in Fig. 1.a) and b), reverse induced current $I_{S}$ will be generated on the braided shield of CUT in case of the injection cable current $I_{i n j}$. That is to say, the induced voltage of inner conductor to braided shield (ground), denoted as $V_{i s g}$ in Fig. 1.c), mainly relies on $I_{S}$ and transfer impedance $Z_{t}$, and then $V_{i s g}$ can be written as

$$
V_{i s g}=I_{s} \cdot Z_{t}
$$

Furthermore, if there is no braided shield in CUT, the induced voltage of inner conductor to ground, $V_{i g}$, is directly derived by

$$
V_{i g}=I_{i n j} \cdot Z_{i n j-i}
$$

And consequently, shield reduction factor $K_{r}$ in $\mathrm{dB}$ can be defined as the ratio of $V_{i s g}$ (with braided shield) to $V_{i g}$ (without braided shield) [5], [6], viz.,

$$
K_{r}(\mathrm{~dB})=20 \cdot \lg \left(\frac{V_{i s g}}{V_{i g}}\right)
$$

Referring to Fig. 1.b), if one considers the case of braided shield to ground having impedance, $Z_{\text {loop }}$, then $I_{s}$ and $I_{i n j}$ are related to this impedance by

$$
I_{\text {inj }} \cdot Z_{\text {inj-s }}-I_{s} \cdot Z_{\text {loop }}=0
$$

Without loss of generality, we can make an assumption that $Z_{\text {inj-i }}=Z_{\text {inj-s }}, Z_{\text {loop }}=R_{s}+j \omega L_{\text {loop }}, Z_{t}=R_{t}+j \omega L_{t}$, and $R_{s} \approx R_{t}$. Combining (1)-(4) yields the following relationships:

$$
K_{r}(\mathrm{~dB})=20 \cdot \lg \left(\frac{Z_{t}}{Z_{\text {loop }}}\right)=20 \cdot \lg \left(\frac{R_{s}+j \omega L_{t}}{R_{S}+j \omega L_{\text {loop }}}\right)
$$

Note that $R_{s}$ and $L_{t}$ are all characteristic parameters associated with CUT. It is clearly counter to the purpose-and to the utility of using shield reduction factor $K_{r}$ to assess the

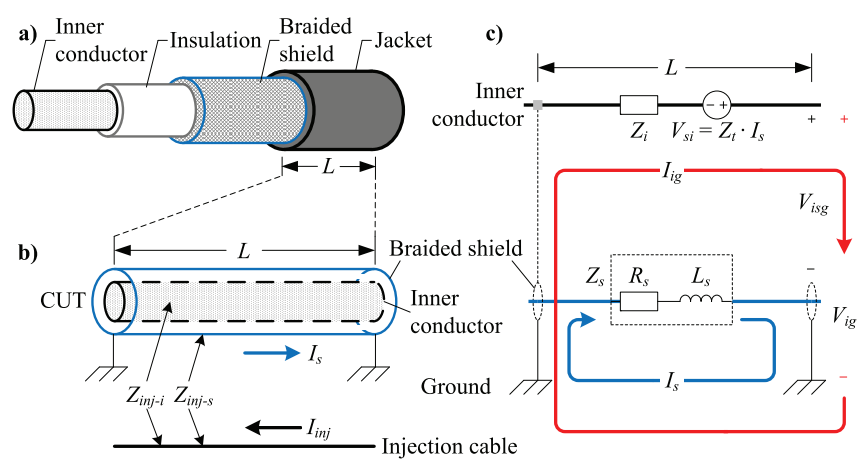

Fig. 1. a) Basic structure of braided-shield cables, b) simple model for two independent parallel cables, and c) equivalent circuit of b). Specially, $L$ is the coupling length, $Z_{i n j-i}$ and $Z_{i n j-s}$ represents the mutual impedance between injection cable and inner conductor, injection cable and braided shield, respectively, and $Z_{s}$ is the impedance of braided shield. 


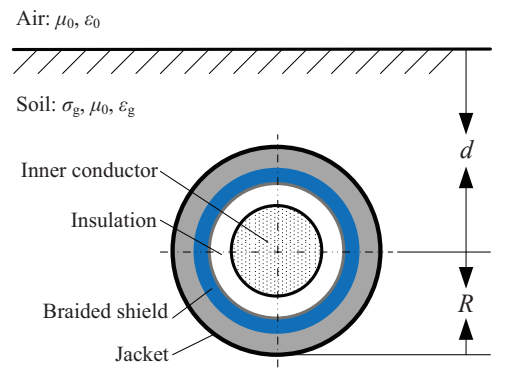

Fig. 2. Braided-shield power cable in the soil. In general, $R$ is the radius of the cable in meters, $d$ is the buried depth in meters, and the soil is charactrized by conductivity $\sigma_{g}$, permeability $\mu_{0}$ and permittivity $\varepsilon_{g}=\varepsilon_{r g} \varepsilon_{0}$.

shielding performance of CUT-if the self inductance $L_{\text {loop }}$ of the braided shield to ground loop is uncertain. In other words, shield reduction factor $K_{r}$ or transfer impedance $Z_{t}$ is sufficient to characterize the suppression of electromagnetic coupling, and in practice, $K_{r}$ can also be used to describe the shielding performance of CUT. For the underground braidedshield power cables, as shown in Fig. 2, the low frequency approximate expression of $L_{\text {loop }}$, proposed by Pollaczek [16], is adopted in GB/T 5441 within the frequency range of 50$800 \mathrm{~Hz}$ and simplified as

$$
\begin{aligned}
& L_{\text {loop }}^{\text {Pollaczek }}(\mathrm{H} / \mathrm{m}) \\
& \left.=\frac{\mu_{0}}{2 \pi} \operatorname{Re}\left[\begin{array}{l}
K_{0}\left(\begin{array}{l}
R \\
\cdot \sqrt{j \omega \mu_{0} \sigma_{g}}
\end{array}\right)-K_{0}\left(\begin{array}{l}
\sqrt{R^{2}+4 d^{2}} \\
\cdot \sqrt{j \omega \mu_{0} \sigma_{g}}
\end{array}\right)+ \\
\left.\int_{-\infty}^{+\infty}\left(\begin{array}{l}
\exp \left(j R u-2 d \sqrt{u^{2}+j \omega \mu_{0} \sigma_{g}}\right) \\
\cdot \mathrm{d} u
\end{array}\right)\right] \\
\approx\left(2 \ln \frac{2}{1.78 R \sqrt{4 \pi \omega \sigma_{g} \times 10^{-7}}}+1\right) \times 10^{-7}
\end{array}\right)\right]
\end{aligned}
$$

where $\operatorname{Re}[\cdot]$ indicates the real part of $Z_{\text {loop }} /(j \omega)$, and $K_{0}$ is the modified Bessel function of the second type of order 0 . It can be seen that (6) is a practical estimation of $L_{\text {loop }}$ without considering the effects of permittivity $\varepsilon_{g}$ and buried depth $d$, and moreover, the usable frequency should satisfy the condition of $f<<\sigma_{g} /\left(2 \pi \varepsilon_{g}\right)$. In [17], a more reasonable expression of $L_{\text {loop }}$, related to the propagation constant $\gamma_{g}=\sqrt{j \omega \mu_{0}\left(\sigma_{g}+j \omega \varepsilon_{g}\right)}$ and buried depth $d$, is given by

$$
\begin{aligned}
& L_{\text {loop }}^{\text {Theethayi }}(\mathrm{H} / \mathrm{m}) \\
& =\operatorname{Re}\left[\frac{\mu_{0}}{2 \pi}\left(\ln \left(\frac{1+r_{g} R}{r_{g} R}\right)+\frac{2}{4+\left(r_{g} R\right)^{2}} e^{-2 d\left|r_{g}\right|}\right)\right]
\end{aligned}
$$

From a practical point of view, $d \rightarrow 0$ in (7) can be used to describe the self inductance $L_{\text {loop }}$ for the case of braidedshield power cables placed on the surface of the ground. As for the others, i.e., a single-braid, with an outer diameter in the $5-15 \mathrm{~mm}$ range, at a height of $50-500 \mathrm{~mm}$ above ground, the average value of $L_{\text {loop }}$ is about $0.955 \mu \mathrm{H} / \mathrm{m}$ [6].

\subsection{Measurement principle and configuration}

According to the definition of $K_{r}$ in (5), the most general measurement setup of SRFM handled in GB/T 5441 is described in Fig. 3. Specifically, fixtures $A_{1}, A_{2}, B_{1}$, and $B_{2}$ are reliably welded to the braided shield of CUT, the distance between $A_{1}$ and $A_{2}, B_{1}$ and $B_{2}$ is $1.02 \mathrm{~m}, 1 \mathrm{~m}$ (i.e., the coupling length $L=1 \mathrm{~m}$ ), respectively. The lead of $A_{1}$ forms a $L \times W$ rectangular loop (see the red line in Fig. 3), and the inductance of that is equal to the self inductance $L_{\text {loop }}$ of the braided shield to ground loop. As per [18] the inductance of $L \times W$ rectangular loop is

$$
L_{\text {loop }}^{L \times W}(\mathrm{H})=\frac{\mu_{0}}{\pi}\left(L \cdot \ln \left(\frac{W}{R}\right)+W \cdot \ln \left(\frac{L}{R}\right)\right)
$$

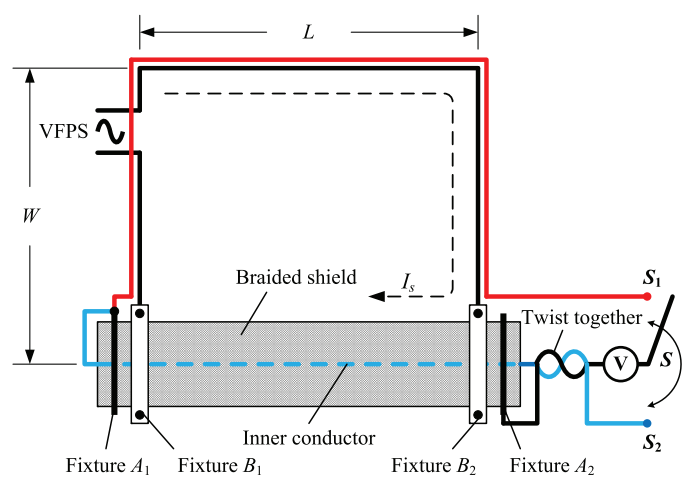

Fig. 3. Standard measurement setup of SRFM for shield reduction factor $K_{r}$ in GB/T 5441.

For the case of $L=1 \mathrm{~m}$, the length of $W$ can be easily derived in terms of (6)-(8). As mentioned before, both $Z_{t}$ and $K_{r}$ are the intrinsic parameters of CUT. However, for the measurement setup, the CUT placed at a large height " $W$ " to the ground will capture more (or radiate more) interference, which will show better $K_{r}$ than the CUT installed closer to the ground. The ability of a cable to radiate or receive is exactly linear with height $W$, while the decrease of $K_{r}$ is only proportional to $\lg W$, and then the $W$ derived by (6) and (8) is a good tradeoff to determine accurately $K_{r}$. As depicted in Fig. 4, the length of $W$ is significantly affected by $\varepsilon_{g}$ and $R$, not the constant value $W=0.4 \mathrm{~m}$ in GB/T 5441. In other words, for the underground braided-shield power cables, the factors such as frequency, soil (conductivity $\sigma_{g}$, permeability $\mu_{0}$, and permittivity $\varepsilon_{g}$ ), buried depth $d$ and cable radius $R$ need to be taken into account to ensure the accurate measurement of $K_{r}$.

Referring to Fig. 3 again, the inner conductor of CUT joins with fixture $A_{1}$ at only one end, and the lead is arranged along the rectangular loop (the red line) in order to provide a measurement terminal $S_{1}$. On the other hand, the inner conductor is twisted together with the end lead of fixture $A_{2}$, which provides another terminal $S_{2}$. Note that the twisted structure is 


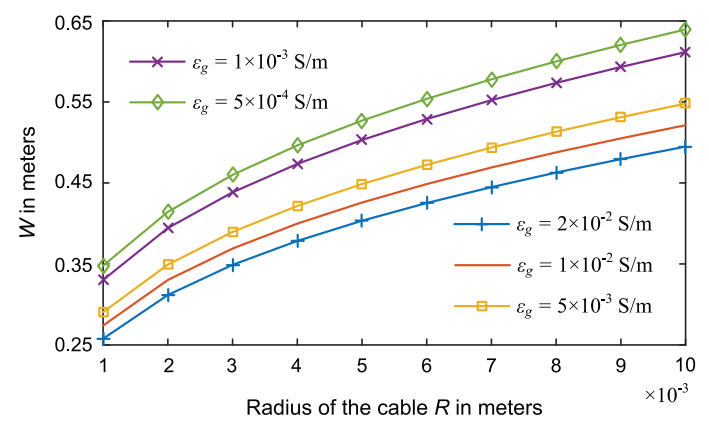

Fig. 4. The length of $W$ for SRFM as a function of radius of CUT for different permittivity.

used to suppress the effect of stray EMF, and there is good insulation between the inner conductor of CUT and the end lead of $A_{2}$. For the case of current excitation generated by VFPS in rectangular loop (see the black line in Fig. 3), voltage $V_{S 1}$ measured by a high dynamic range sensor with switch $S$ connecting to $S_{1}$ indicates the induced voltage $V_{i g}$ without braided shield (see Fig. 1.c)), while the voltage $V_{S 2}$ with $S$ connecting to $S_{2}$ represents $V_{i s g}$ with braided shield. Obviously, shield reduction factor $K_{r}$ can be calculated as

$$
K_{r}=\frac{V_{i s g}}{V_{i g}}=\frac{V_{S 2}}{V_{S 1}}
$$

It is worth noting that VFPS is directly loaded onto the braided shield of CUT, and it is uncertain how many amperes will have to be supplied by the source to ensure that the measurement of sensor has a sufficient dynamic range. Moreover, it is difficult to avoid ground-loop and stray EMF effects on broadband sensor signal typically below $100 \mathrm{kHz}$.

In view of this, the improved measurement configuration for SRFM is shown in Fig. 5. Two cables with the same length are placed in parallel, and a pair of fixtures are reliably welded to both sides of the braided shield of CUT. The leads of fixture form a $L \times W$ rectangular loop, and the inner-conductor of $P_{4}$ is welded to the adjacent fixture. The inner-conductor of $P_{3}$ directly connects with the braided shield of injection cable, and leads of inner-conductor and braided shield in $P_{1}$ and $P_{2}$ joint with coaxial connector, respectively. VNA (Agilent Technologies E5061B) and power splitter are essential for the measurements, where VNA provides gain-phase test port $\left(5 \mathrm{~Hz}\right.$ to $30 \mathrm{MHz}, Z_{\text {in }}=1 \mathrm{M} \Omega$ or $50 \Omega$ ) that can be used not only as a broadband power source but also as a highprecision sensor with a dynamic range of $\geq 90 \mathrm{~dB}$. Specifically, LF-OUT and R-ch receiver of VNA connects with port 1 and 2 of power splitter, respectively. Port 3 of power splitter is connected to $P_{1}$ while T-ch receiver of VNA connects with $P_{2}$.

In the simplest case, when $S$ is on, there will be induced current in the $L \times W$ rectangular loop, the T/R parameter measured by VNA represents the ratio of induced voltage $V_{\text {isg }}$ to $\mathrm{R}$-ch receiver in case of effective grounding of the braided shield, and is denoted as $20 \cdot \lg \left(T_{o n} / R\right)$. Similarly, $S$ is off, the $\mathrm{T} / \mathrm{R}$ parameter measured by VNA indicates the ratio of in- duced voltage $V_{i g}$ to R-ch receiver with no braided shield, and is denoted as $20 \cdot \lg \left(T_{o f f} / R\right)$. Then $K_{r}$ can be derived by

$$
K_{r}(\mathrm{~dB})=20 \cdot \lg \frac{T_{\text {on }}}{R}-20 \cdot \lg \frac{T_{\text {off }}}{R}=20 \cdot \lg \frac{T_{\text {on }}}{T_{\text {off }}}
$$

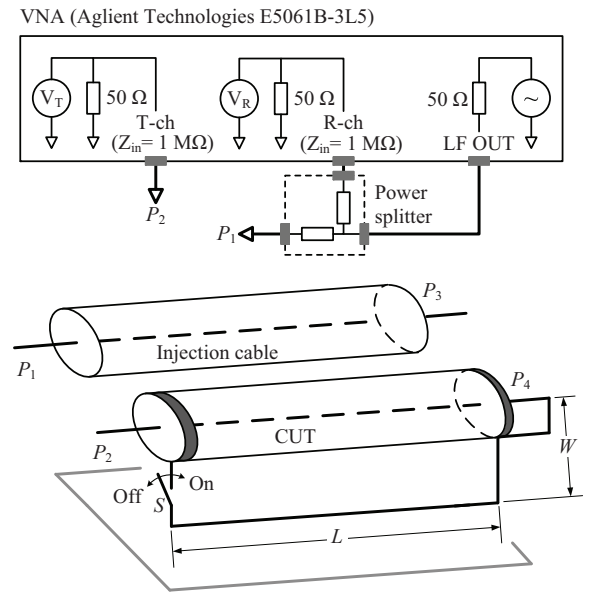

Fig.5. Conceptual view of the improved measurement configuration for SRFM, where $P_{1}-P_{4}$ indicate the port of injection cable and CUT.

Referring to Fig. 5, the LF-OUT is set to $0 \mathrm{dBm}$, the braided shield resistance $R_{S}$ of injection cable and CUT is several $\mathrm{m} \Omega$ s, the input impedance $Z_{i n}$ for T-ch and R-ch receiver should be set to $1 \mathrm{M} \Omega$. It means that $Z_{\text {in }} \gg R_{s}$, i.e., $R$ in $\mathrm{T} / \mathrm{R}$ measured by VNA is constant value for a given frequency, no matter if $S$ is on or off. Furthermore, it is worth noting that the injection cable and CUT are two relatively independent test loops, there is no ground-loop effect especially for the T-ch receiver. With regard to stray EMF, it has an influence on both measurements, which indicates the stray EMF effects (denoted as $20 \cdot \lg \left(T_{E M F} / R\right)$ ) can be eliminated by simple mathematical operations, namely

$$
\begin{aligned}
& K_{r}(\mathrm{~dB}) \\
& =\left\{\begin{array}{l}
\left(20 \cdot \lg \frac{T_{\text {on }}}{R}-20 \cdot \lg \frac{T_{E M F}}{R}\right) \\
-\left(20 \cdot \lg \frac{T_{o f f}}{R}-20 \cdot \lg \frac{T_{E M F}}{R}\right)
\end{array}\right\}=20 \cdot \lg \frac{T_{o n}}{T_{o f f}}
\end{aligned}
$$

\section{RESUltS AND DisCUSSION}

Fig. 6 shows the measurement setup to assess the shielding performance of braided-shield power cable from 25 $\mathrm{Hz}$ to $1 \mathrm{MHz}$, where CUT is CJPF96/SC $2 \times 2.5 \mathrm{~mm}^{2}$ (Changzhou Marine Cable Co., Ltd., China), $W=0.12 \mathrm{~m}$ in case of $L=1 \mathrm{~m}$ and $L_{\text {loop }}=0.955 \mu \mathrm{H} / \mathrm{m}$. Note that although these results have been discussed in detail in [7], the re-explanation gives useful insight for understanding the shield reduction factor $K_{r}$ and the related method (SRFM).

As previously mentioned in (10), $K_{r}$ can be easily derived by $\mathrm{T} / \mathrm{R}$ parameters measured by VNA, and consequently $Z_{t}$ 

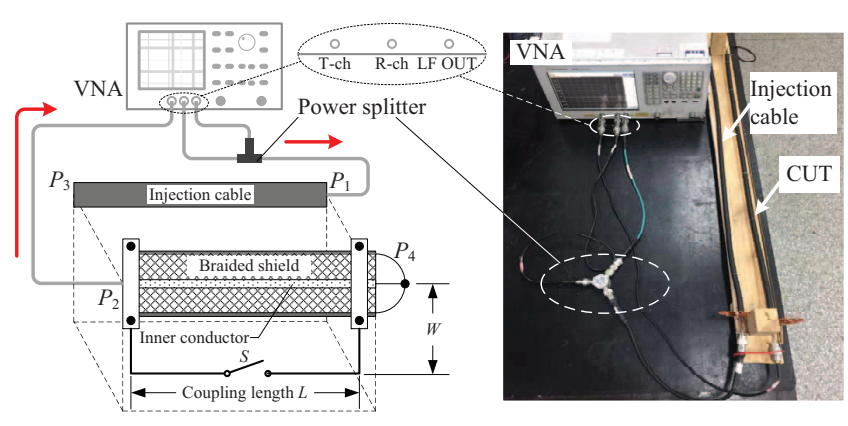

Fig. 6. Measurement test bench.
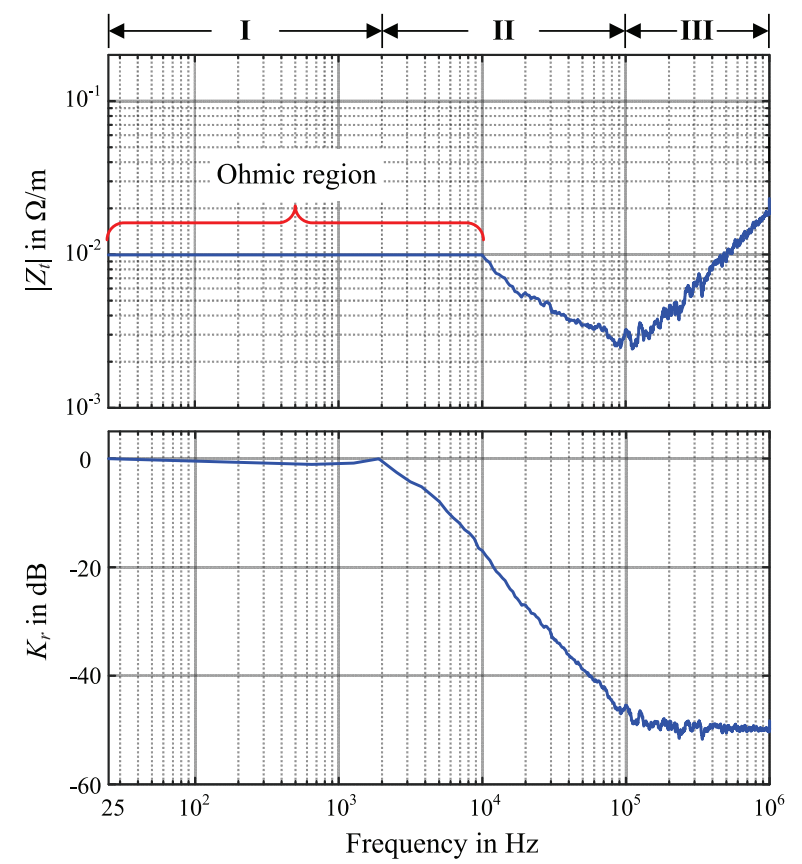

Fig. 7. Frequency-dependent magnitude of transfer impedance $Z_{t}$ (Upper) and shield reduction factor $K_{r}$ (Lower).

is calculated according to (5). For the sake of convenience, these values are divided into three basic frequency ranges, i.e., domain I, II, and III as shown in Fig. 7. At lower frequencies (domain I below $2 \mathrm{kHz}$ ), $R_{s} \gg \omega L_{t}, R_{s}>\omega L_{\text {loop }}$, and (5) can be simplified as

$$
K_{r}(\mathrm{~dB}) \approx 20 \cdot \lg \left(\frac{R_{s}}{R_{S}+j \omega L_{\text {loop }}}\right)
$$

Referring to the ohmic region shown in Fig. 7, $Z_{t}$ is regarded as equal to $R_{S} \approx 9.93 \mathrm{~m} \Omega / \mathrm{m}$, which has been verified by the direct measurement of $R_{S}$ with the use of milliohmmeter (Tonghui TH2521 at $1 \mathrm{kHz}$ ). Obviously, $K_{r}$ tends to a constant value approaching $0 \mathrm{~dB}$, which indicates that the braided shield of CUT offers relatively poor shielding performance below $2 \mathrm{kHz}$.

Regarding domain II from $2 \mathrm{kHz}$ to $100 \mathrm{kHz}, Z_{\text {loop }}$ relies on $\omega L_{\text {loop }}$, the corresponding range is approximately from 0.012
$\Omega$ to $0.6 \Omega$, and (5) is written as

$$
K_{r}(\mathrm{~dB}) \approx 20 \cdot \lg \left(\frac{R_{s}+j \omega L_{t}}{j \omega L_{\text {loop }}}\right)
$$

It can be seen that $K_{r}$ is negatively correlated with frequency, and the experimental value of $K_{r}$ shown in Fig. 7 also verifies the similar frequency-dependent behavior, i.e., $K_{r}$ decreases with increasing frequency. Referring to $\omega L_{t}$, the minimum value is about $1.9 \mathrm{~m} \Omega$ at $100 \mathrm{kHz}$, and meanwhile, the best shielding performance of $K_{r}=-50 \mathrm{~dB}$ can be obtained for the CUT. Considering the equivalent model of series resistance $R_{s}$ and inductance $L_{t}$ shown in Fig. 1.c), $Z_{t}$ still depends on $R_{s}$, and $Z_{t}$ is negatively correlated with frequency from 10 $\mathrm{kHz}$ to $100 \mathrm{kHz}$ which has also been verified experimentally in Fig. 7. More precisely, as discussed in [19], the frequencydependent behavior of $Z_{t}$ mainly relies on the scattering part (denoted as $Z_{d}$ ) of $R_{s}$, namely

$$
Z_{t} \approx Z_{d}=R_{s} \frac{m_{\delta}}{\sinh \left(m_{\delta}\right)}
$$

where $m_{\delta}$ is positively correlated with $d / \delta, d$ is the thickness of single braided wire and $\delta$ is the skin depth. As frequency increases, the skin depth $\delta$ decreases, and the corresponding $m_{\delta}$ increases. Specifically, if $m_{\delta} \leq 1$, then $\sinh \left(m_{\delta}\right) \approx m_{\delta}$, i.e., $f \leq 10 \mathrm{kHz}$, and $Z_{t} \approx R_{S}$ as shown in the ohmic region. Otherwise, if $m_{\delta}>1$, then $\sinh \left(m_{\delta}\right)>m_{\delta}$, and consequently $Z_{t}$ decreases with increasing frequency in terms of (14), which can be used to explain the behavior of $Z_{t}$ in the non-ohmic region of domain II.

Regarding domain III above $100 \mathrm{kHz}$, the inductance component in $Z_{t}$ is gradually significant, and $Z_{t}$ increases linearly with the frequency. Therefore, (5) can also be further simplified as

$$
K_{r}(\mathrm{~dB}) \approx 20 \lg \left(\frac{L_{t}}{L_{\text {loop }}}\right)
$$

and $K_{r}$ keeps a constant value $(50 \mathrm{~dB})$ related to the material and geometric parameters of the braided shield.

And yet, for the frequency from $10 \mathrm{kHz}$ to first $<\lambda / 2$ ( $\lambda$ being the wavelength) resonance, a simple empirical formula derived in [5] can be used to assess $K_{r}$, i.e.,

$$
K_{r}(\mathrm{~dB})==-20 \cdot \lg \left(1+\frac{6 f(\mathrm{MHz})}{Z_{t}(\Omega / \mathrm{m})}\right)
$$

\section{CONCLUSIONS}

This work proposes an improved shield reduction factor method for the electromagnetic shielding performance measurement of braided-shield power cables at low frequency region from $25 \mathrm{~Hz}$ to $1 \mathrm{MHz}$. Test cell, consisting of two parallel braided-shield cables and a $L \times W$ rectangular loop, is simplified according to the definition of the shield reduction factor. With and without shield connecting to the $L \times W$ rectangular loop, the gain (T/R) parameters, measured by VNA, can be used for the calculation of both shield reduction factor and transfer impedance. It is worth noting that $\mathrm{R}$-ch receiver of VNA is set to $1 \mathrm{M} \Omega$, the R parameters fed back over power 
splitter are the same whether the braided shield connects to the rectangular loop or not, and furthermore, the measurement configuration also provides more effective countermeasures to avoid the effects of ground-loop and stray EMF.

The present study mainly highlights the implementation of measurements from an engineering perspective, and the corresponding principle. The comparative discussion between transfer impedance and shield reduction factor indicates that shield reduction factor can provide more intuitive evaluation of shielding behavior of braided-shield power cables at low frequency regimes.

A promising point of further work is that the extension of SRFM can be used as an indicator to improve the shielding performance of braided-shield cables, e.g., the material and geometric parameters of the braided shield may be optimized by the finite element method or, perhaps, other relevant numerical methods and simulation techniques.

\section{REFERENCES}

[1] Hanigovszki, N., Spiazzi, G., Blaabjerg, F. (2006). An EMC evaluation of the use of unshielded motor cables in AC adjustable speed drive applications. IEEE Transactions on Power Electronics, 21 (1), 273-281.

[2] Baklezos, A.T., Nikolopoulos, C.D., Katsouris, A.G., Koutantos, G.I., Capsalis, C.N. (2016). Electromagnetic emission modeling in case of shielded cabling with respect to the ground dielectric properties. IEEE Transactions on Electromagnetic Compatibility, 58 (6), 16941700.

[3] Yousaf, J., Amin, M., Iqbal, S. (2013). Investigation of low frequency emissions due to interfacing cables in RE 102. In International Conference on Space Science and Communication (IconSpace), 1-3 July 2013. Melaka, Malaysia: IEEE, 65-69.

[4] Mariscotti, A. (2007). Induced voltage calculation in electric traction systems: simplified methods, screening factors, and accuracy. IEEE Transactions on Intelligent Transportation Systems, 12 (1), 201-210.

[5] Mardiguian, M. (2012). Simple method for predicting a cable shielding factor, based on transfer impedance. Interference Technology, https://interferencetechnology.com/simple-method-forpredicting-a-cable-shielding-factor-based-on-transferimpedance/.

[6] Mardiguian, M. (2014). Controlling Radiated Emissions by Design, (3rd ed.). New York, USA: Springer, 291301.

[7] Hu, P., Zhou, Z.Z., Li, J.P., Zhou, X., Sheng M.J., Li, P., Zhou, Q. (2019). Measurement techniques for electromagnetic shielding behavior of braided-shield power cables: an overview and comparative study. Measurement Science Review, 19 (5), 213-221.

[8] International Electrotechnical Commission. (2013). Metallic communication cable test methods - Part 4-3: Electromagnetic compatibility (EMC) - Surface trans- fer impedance - Triaxial method. IEC 62153-4-3: 2013. Geneva.

[9] Mushtaq, A., Frei, S. (2016). Alternate methods for transfer impedance measurements of shielded HVcables and $\mathrm{HV}$-cable-connector systems for EV and $\mathrm{HEV}$. International Journal of $R F$ and Microwave Computer-Aided Engineering, 26 (4), 359-366.

[10] International Electrotechnical Commission. (2017). Metallic cables and other passive components test methods - Part 4-6: Electromagnetic compatibility (EMC) Surface transfer impedance - line injection method. IEC 62153-4-6:2017. Geneva.

[11] Mushtaq, A., Frei, S., Siebert, K., Barenfangeret, J. (2013). Analysis of shielding effectiveness of HV cable and connector systems used for electric vehicles. In International Conference on Electromagnetic Compatibility (EMC Europe), 2-6 September 2013. Brugge, Belgium: IEEE, 241-246.

[12] Mushtaq, A., Frei, S. (2016). Transfer impedance simulation and measurement methods to analyse shielding behaviour of $\mathrm{HV}$ cables used in Electric-Vehicles and Hybrid-Electric-Vehicles. Advances in Radio Science, (14), 139-145.

[13] Wulf, M.D., Wouters, P., Sergeant, P., Dupre, L., Hoferlin, E., Jacobs, S., Harlet, P. (2007). Electromagnetic shielding of high-voltage cables. Journal of Magnetism and Magnetic Materials, (316), 908-911.

[14] Zhu, K., Lee W.K., Pong P.W.T. (2017). Energizationstatus identification of three-phase three-core shielded distribution power cables based on non-destructive magnetic field sensing. IEEE Sensors Journal, (19), 74057417.

[15] National Standards of the People's Republic of China. (2016). Test Methods for Communication Cable. GB/T 5441-2016. Beijing.

[16] Pollaczek, F. (1931). Sur le champ produit par un conducteur simple infiniment long parcouru par un courant alternatif. Revue Generale De l Electricite, (29), 851867.

[17] Theethayi, N., Thottappillil, R., Paolone, M., Nucci, C.A., Rachidi F. (2007). External impedance and admittance of buried horizontal wires for transient studies using transmission line analysis. IEEE Transactions on Dielectrics and Electrical Insulation, 14 (3), 751-761.

[18] Ott, H.W. (2009). Electromagnetic Compatibility Engineering. Hoboken, NJ, USA: John Wiley \& Sons, Inc., 767-769.

[19] Tesche, F.M., Ianoz, M.V., Karlsson, T. (2009). EMC Analysis Methods and Computational Models, (Chinese ed.). Beijing, China: Beijing University of Posts and Telecommunications Press, 283-313.

Received July 6, 2020. Accepted October 12, 2020. 